\title{
Assessment of Erythrobacter Species Diversity through Pan-Genome Analysis with Newly Isolated Erythrobacter sp. 3-20A1M
}

\author{
Sang-Hyeok Cho' ${ }^{1}$, Yujin Jeong ${ }^{1}$, Eunju Lee', So-Ra Ko ${ }^{3}$, Chi-Yong Ahn ${ }^{3}$, Hee-Mock $\mathrm{Oh}^{3}$, \\ Byung-Kwan $\mathrm{Cho}^{1,2 *}$, and Suhyung Cho ${ }^{1,2 *}$ \\ ${ }^{1}$ Department of Biological Sciences, Korea Advanced Institute of Science and Technology, Daejeon 34141, Republic \\ of Korea \\ ${ }^{2}$ KI for the BioCentury, Korea Advanced Institute of Science and Technology, Daejeon 34141, Republic of Korea \\ ${ }^{3}$ Biological Resource Center, Korea Research Institute of Bioscience and Biotechnology, Daejeon 34141, Republic of Korea
}

Erythrobacter species are extensively studied marine bacteria that produce various carotenoids. Due to their photoheterotrophic ability, it has been suggested that they play a crucial role in marine ecosystems. It is essential to identify the genome sequence and the genes of the species to predict their role in the marine ecosystem. In this study, we report the complete genome sequence of the marine bacterium Erythrobacter sp. 3-20A1M. The genome size was 3.1 Mbp and its GC content was $\mathbf{6 4 . 8 \%}$. In total, 2998 genetic features were annotated, of which $\mathbf{2 8 8 2}$ were annotated as functional coding genes. Using the genetic information of Erythrobacter sp. 3-20A1 M, we performed pangenome analysis with other Erythrobacter species. This revealed highly conserved secondary metabolite biosynthesis-related COG functions across Erythrobacter species. Through subsequent secondary metabolite biosynthetic gene cluster prediction and KEGG analysis, the carotenoid biosynthetic pathway was proven conserved in all Erythrobacter species, except for the spheroidene and spirilloxanthin pathways, which are only found in photosynthetic Erythrobacter species. The presence of virulence genes, especially the plant-algae cell wall degrading genes, revealed that Erythrobacter sp. 3-20A1M is a potential marine plant-algae scavenger.

Keywords: Erythrobacter, whole-genome sequencing, pan-genome analysis, secondary metabolites

Received: December 31, 2020 Accepted: February 2, 2021

First published online: February 3, 2021

*Corresponding authors S. Cho

Phone: +82-42-350-2660

Fax: +82-42-350-5620

E-mail: shcho95@kaist.ac.kr

B.-K. Cho

E-mail: bcho@kaist.ac.kr

Supplementary data for this paper are available on-line only at http://jmb.or.kr.

pISSN 1017-7825 eISSN 1738-8872

Copyright(C) 2021 by The Korean Society for Microbiology and Biotechnology

\section{Introduction}

Among marine microorganisms, Erythrobacter species contribute to carbon distribution in the ocean [1]. While diverse microorganisms participate in the carbon cycle via autotrophic inorganic carbon fixation or heterotrophic organic carbon redistribution, photoheterotrophic Erythrobacter species potentially mediate both organic and inorganic carbon cycles [2-4]. Photoheterotrophic bacteria called aerobic anoxygenic phototrophic bacteria (AAPB), which include Erythrobacter species, such as E. litoralis and E. longus, harvest light energy using photosynthetic gene clusters (PGCs) [5]. The non-AAPB strains were discovered after the establishment of the Erythrobacter genus, in which photosynthetic marine bacteria were classified [6]. The photosynthetic ability of Erythrobacter species is shared with purple phototrophic bacteria, which are phylogenetically closely related [7].

Erythrobacter, namely the red (erythro-) bacteria (-bacter), are well-known carotenoid-producing microorganisms [8]. Carotenoids are a family of bioactive, yellow to orange colored, pigment compounds used medicinally for their antioxidant properties, disease risk-reducing benefits, and enhancement of immune functions [9]. Carotenoid derivatives are produced from terpenoid backbone precursors, such as geranyl-geranyl pyrophosphate, which is also produced from pyruvate and glyceraldehyde 3-phosphate via the terpene biosynthetic pathway. E. longus, for example, produces various carotenoids, including $\beta$-carotene, zeaxanthin, nostoxanthin, caloxanthin, $\beta$ cryptoxanthin, rubixanthin, bacteriorubixanthin, anhydrorhodovibrin, and erythroxanthin [8]. Erythrobacter species have different carotenoid production profiles depending on their genetic composition. In particular, AAPB species produce unique carotenoids named spheroidene or spirilloxanthin, which are utilized as photosynthetic pigments $[7,10]$.

Owing to the development of next-generation sequencing techniques and subsequent bioinformatics tools, it is now possible to obtain massive amounts of genetic information. Based on genetic information, the phylogeny of new taxa can be identified more precisely. In addition, metabolite profiles can be predicted based on reconstructed 
metabolic pathways, and the ecological niche can be studied based on genetic profiles [11,12]. In this study, we sequenced the genome of Erythrobacter sp. 3-20A1M. We report the complete genome sequence of this species and its genetic characteristics through gene annotations. Additionally, the distinguishable characteristics of Erythrobacter sp. 3-20A1M were identified by comparing its secondary metabolite profile with that of other Erythrobacter species through pan-genome analysis.

\section{Materials and Methods}

Isolation and Scanning Electron Microscopy

Erythrobacter sp. 3-20A1M was isolated from the southern sea of GeoJe, South Korea $\left(34^{\circ} 46^{\prime} \mathrm{N}, 128^{\circ} 46^{\prime} \mathrm{E}\right)$, in August 2016 and deposited in the Korea Collection for Type Cultures (KCTC; Accession \#, KCTC 18715P). The specimen was cultured in marine broth 2216 (BD Difco, USA) or marine agar at $25^{\circ} \mathrm{C}$. For scanning electron microscopy (SEM), Erythrobacter sp. 3-20A1M was cultured in marine broth and centrifuged at 4,000 rpm at $4{ }^{\circ} \mathrm{C}$. The bacterial cell pellet was resuspended in a $2.5 \%$ paraformaldehyde-glutaraldehyde mixture buffered with $0.1 \mathrm{M}$ phosphate ( $\mathrm{pH}$ 7.2). The sample was fixed in the solution for 2 hours, post-fixed in $1 \%$ osmium tetroxide in the same buffer for 1 hour, dehydrated in graded ethanol, which was then substituted with isoamyl acetate. They were then dried at a critical point in $\mathrm{CO}_{2}$. Finally, the samples were sputtered with gold in a sputter coater (SC502, Polaron) and observed using a scanning electron microscope and FEI Quanta 250 FEG (FEI, USA).

\section{Genome Sequencing and Genome de novo Assembly}

Genomic DNA of Erythrobacter sp. 3-20A1M was extracted using the Wizard Genomic DNA Purification Kit (Promega) following the manufacturer's protocol. The quality of the extracted genomic DNA was assessed by a NanoDrop TM2000 (Thermo Fisher Scientific, USA) for a UV absorbance ratio (260:280) of 2 and inspection using 1\% agarose gel electrophoresis. A genome sequencing library with an insert size of 550 bp was prepared using the TruSeq Nano DNA Library Prep Kit (Illumina, USA) following the manufacturer's protocol. The prepared genome sequencing library was sequenced using a 250-cycle paired-end reaction on the Illumina MiSeq platform. Raw sequencing reads were subjected to PlasmidSeeker to detect native plasmids [13]. Sequencing data were processed using CLC Genomics Workbench 6.5.1. software (CLC Bio, Denmark). PhiX, adapters, and quality trimmed reads were used for de novo genome assembly (word size $=24$, bubble size = automatic, and mapping option $=$ map reads back to contigs (slow)). The assembled genome sequence was assessed using Benchmarking Universal Single-Copy Orthologs (BUSCO). The complete genome sequence was deposited in GenBank under accession number CP045200.

\section{Phylogenetic Analysis}

Genomic sequences of each strain used for phylogenetic analysis were downloaded from the NCBI Genome Portal (Table S1). The phylogenetic tree was reconstructed using the Up-to-date Bacterial Core Gene (UBCG) analysis pipeline [14]. Randomized Axelerated Maximum Likelihood (RAxML) was used to generate the phylogenetic tree from the calculated distance data [15]. 16s rRNA based phylogeny analysis was conducted using MEGA-X [16]. The evolutionary history was inferred using the Neighbor-Joining method and the evolutionary distances were computed using the p-distance method.

\section{Gene Annotation and Secondary Metabolite Biosynthesis Gene Prediction}

The de novo assembled genome sequence of Erythrobacter sp. 3-20A1M was annotated using the NCBI Prokaryotic Genome Annotation Pipeline [17]. Subsequently, the amino acid sequences were extracted from the annotated coding genes and searched against KEGG Orthology (KO) ID, Clusters of Orthologous Groups (COG), and Gene Ontology (GO) using EggNOG-mapper (version 2) [18-21].

\section{Pan-Genome Analysis and Prediction of Secondary Metabolite Clusters}

For the pan-genome analysis of Erythrobacter species, the pan-genome analysis pipeline (PGAP) (version 1.2.1) was used [22]. Ortholog clusters were organized using the coding sequences (CDSs) of each genome with the gene family (GF) method under default parameters (E-value: 1e10, score: 40; identity: 50; coverage: 50). To generate additional COG input data, all Erythrobacter genomes used were functionally annotated with EggNOG-Mapper (version 2) with their amino acid sequences. Secondary metabolite biosynthetic gene clusters were predicted using antiSMASH (version 5.2.0) [23]. The assembled genome sequence was assessed using BUSCO [24].

\section{Results and Discussion}

\section{Phenotypic and Genotypic Identification of Erythrobacter sp. 3-20A1M}

First, the morphological features of Erythrobacter sp. 3-20A1M were observed. As the name suggests, Erythrobacter is a bacterium with a yellow to orange color, and the cell pellet of Erythrobacter sp. 3-20A1M also had an orange color. The cell shape of the bacteria was observed using SEM (Fig. 1A). The bacteria had an irregular cell shape under the SEM. The shape ranged from spherical to rod-shaped. The length and breadth of the cell was $0.5-2.0 \mu \mathrm{m}$ and $0.5 \mu \mathrm{m}$, respectively. It has been reported that Erythrobacter species use binary fission for reproduction; however, irregular divisions, such as budding or Y cell division, are also seen at higher growth rates [10]. In addition, a glue-like substance was identified on the cell surface in SEM images, which confirmed that Erythrobacter sp. 3-20A1M forms a biofilm.

We performed genome sequencing and genome assembly to analyze the genetic component of Erythrobacter sp. 
A

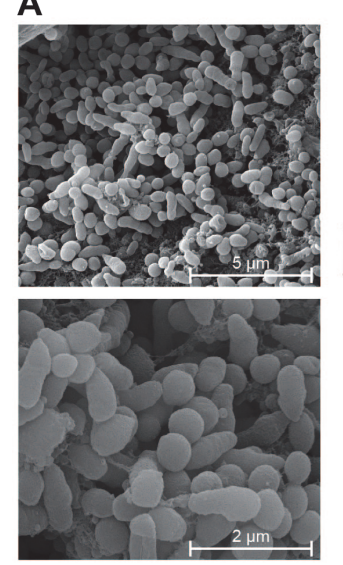

B

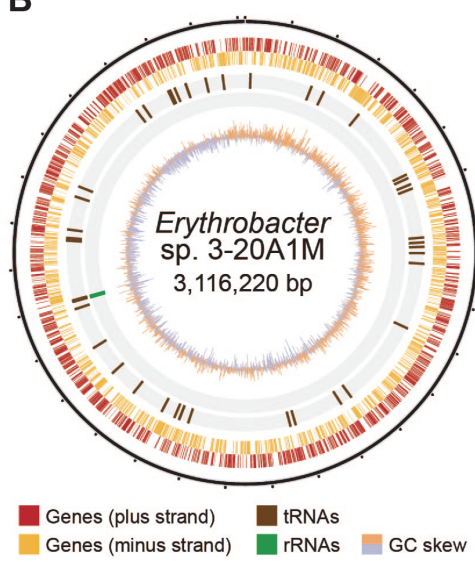

C

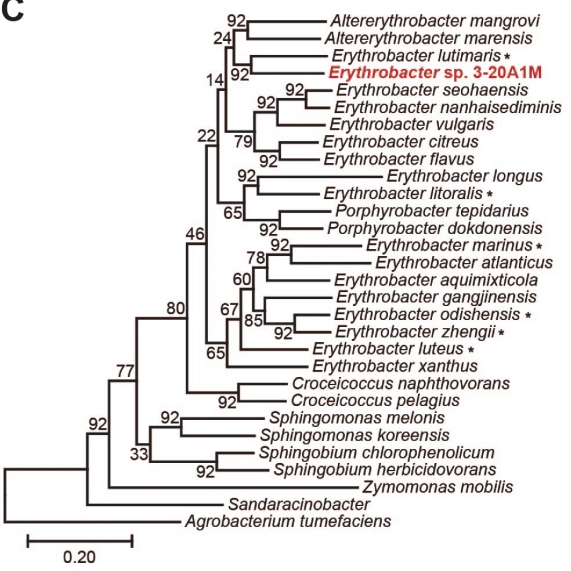

Fig. 1. Identification of Erythrobacter sp. 3-20A1M. A. Scanning electron micrograph of Erythrobacter sp. 3-20A1M. B. Circular representation of the Erythrobacter sp. 3-20A1M complete genome. From the outside to the center: genome (black, ticks every $100 \mathrm{Kbp}$ ), genes on the plus strand (red), genes on the minus strand (yellow), tRNA (brown), rRNA (green), and the GC skew (orange and light purple). C. Phylogenetic analysis of Erythrobacter sp. 3-20A1M and 29 closely related taxa, performed based on their core genes. Agrobacterium tumefaciens was selected as the outgroup. The tree is drawn to scale, with branch length units equivalent to those of evolutionary distances used to infer the phylogenetic tree. The evolutionary distances were provided by Up-to-date Bacterial Core Gene (UBCG) and plotted by Randomized Axelerated Maximum Likelihood (RAxML).

3-20A1M. The genome assembly provided a complete genome of $3.1 \mathrm{Mbp}$ (Fig. 1B). The quality of the assembled genome was assessed using BUSCO, and duplicated or fragmented orthologs were not found (Table S2) [24]. The DNA GC content of the genome was $64.8 \%$. The assembled genome sequence was annotated using the NCBI Prokaryotic Genome Annotation Pipeline [17]. As a result, 2998 features were annotated (Table 1 and Supplementary Dataset S1). Of the 2946 annotated CDSs, 2830 functional CDSs were identified, excluding 116 pseudogenes. The remaining 52 features were RNA genes, including genes of three complete rRNAs (5S, 16S, and 23S), 45 tRNAs, and four non-coding RNAs.

To identify and confirm the evolutionary relationship of the newly sequenced bacterium, we performed a phylogenetic analysis based on core Erythrobacter sp. 3-20A1M genes with 16 other Erythrobacter species and 12 other species from the order Sphingomonadales, which are closely related to Erythrobacter (Fig. 1C and Table S1). Agrobacterium tumefaciens was included as an outgroup for the analysis of 30 species. The evolutionary distance was calculated using UBCG and the phylogenetic tree was visualized using RAxML [14, 15]. Erythrobacter, within the order Sphingomonadales and the family Sphinogomonadaceae, were grouped under branches in close proximity, and Erythrobacter sp. 3-20A1M formed a single phylogenetic cluster with E. lutimaris. In addition, Porphyrobacter within the same order and family as Erythrobacter, was close to Erythrobacter species in phylogenetic distance. However, Sphingomonas, classified in the same order and family as Erythrobacter, and Croceicoccus, classified in the order Sphingomonadales and the family Erythrobacteraceae, were far from Erythrobacter species in phylogenetic distance $[25,26]$. The 16s rRNA sequence-based phylogeny analysis also supports the novelty of the newly identified Erythrobacter specimen (Fig. S1).

\section{Functional Categorization}

The functional composition of the encoded genetic features in Erythrobacter sp. 3-20A1M was categorized according to $\mathrm{KO}, \mathrm{COG}$, and GO [18-21]. A total of 2882 coding genes were assigned to $1731 \mathrm{KO}$ IDs, $2481 \mathrm{COG}$ functions, and 685 GO terms (Fig. 2 and Table S3). In the KEGG analysis, genes involved in carbohydrate and amino acid metabolism were abundant, similar to most microorganisms. In particular, genes related to the metabolism of cofactors and vitamins were ranked high, reflecting the higher carotenoid synthesis level of

Table 1. Gene annotation statistics.

\begin{tabular}{cc}
\hline Features annotated & Erythrobactersp. 3-20A1M \\
\hline Coding sequences (CDSs) & 2,946 \\
Functional CDSs & 2,830 \\
Pseudogenes & 116 \\
RNA genes & 52 \\
rRNAs & $1,1,1(5 \mathrm{~S}, 16 \mathrm{~S}, 23 \mathrm{~S})$ \\
tRNAs & 45 \\
ncRNAs & 4 \\
Total annotated features & 2,998 \\
\hline
\end{tabular}



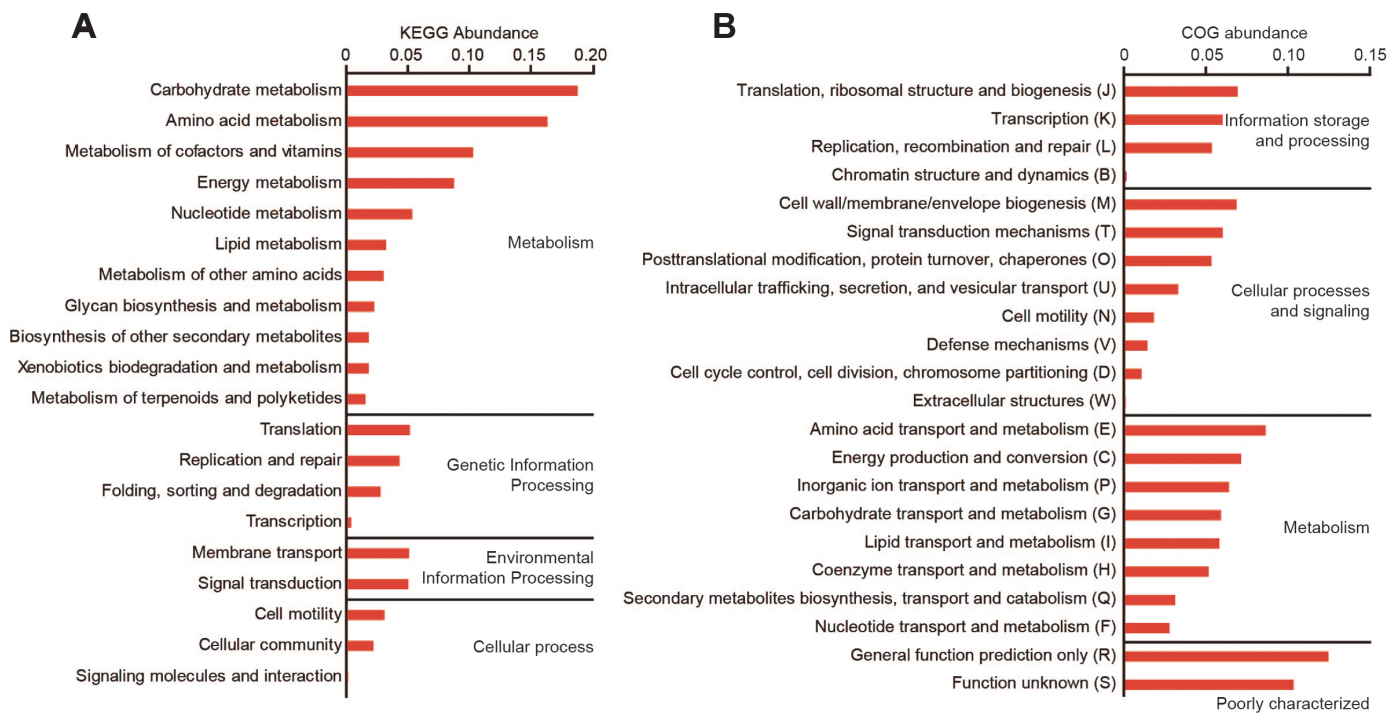

Fig. 2. KEGG pathway analysis and COG analysis. EggNOG-mapper analysis of 2882 Erythrobacter sp. 3-20A1M coding genes. A. KEGG Orthology categorized 1731 genes. B. Clusters of Orthologous Groups categorized 2481 genes.

Erythrobacter producing its red-orange color (Fig. 2A). Moreover, genes related to membrane transport and signal transduction, involved in environmental information processing, were present. This suggests that Erythrobacter sp. 3-20A1M may play a role in the exchange of cellular metabolites with other microorganisms. In COG analysis, the genes for amino acid transport and metabolism (F); energy production and conversion (C); and translation, ribosomal structure, and biogenesis ( $\mathrm{J}$ ) were abundant, except for poorly categorized categories (R and S) (Fig. 2B). It also has a high percentage of genes involved in inorganic ion transport and metabolism.

\section{Pan-Genome Analysis of 17 Erythrobacter Species}

We conducted pan-genome analysis of 17 Erythrobacter species, including Erythrobacter sp. 3-20A1M (Table S1). Their genome sizes varied between a minimum of $2.6 \mathrm{Mbp}$ in E. nanhaisediminis to a maximum of 4.4 Mbp in E. xanthus (Fig. 3A), while their GC content ranged from $57.4 \%$ to $67.2 \%$.

In the PGAP, the pan-genome size of Erythrobacter species increased with an increasing number of species, implying that the pan-genome of Erythrobacter species belongs to the open pan-genome category (Fig. 3B) [22, 27]. A total of 1065 Erythrobacter genes were conserved across the 17 species, and thus were denoted as core genes (Fig. 3C). The core genes of Erythrobacter species accounted for $26.3 \%$ to $40.2 \%$ of all genes, and Erythrobacter sp. $3-20 \mathrm{~A} 1 \mathrm{M}$ had a core-genome ratio of $35.2 \%$. At least two species maintained 4750 that were classified as dispensable genes. The number of unique genes in a species varied from 217 to 1312, with E. xanthus having the largest genome size and the largest number of unique genes.

We compared the COG category-based variability in the distributions of PGAP classifications, including core, dispensable, and specific (Fig. 3D). As a result, the core gene ratio was the highest with COG functions related to cell cycle control, cell division, and chromosome partitioning (D). Interestingly, the second-highest core gene proportion was found in the secondary metabolite biosynthesis, transport, and catabolism (Q) COG function. Since Erythrobacter species are known to produce carotenoids, it was inferred that the genes related to carotenoid biosynthesis might have been attributed to the high conservation ratio of the secondary metabolite related gene functions.

In the KEGG analysis, Erythrobacter sp. 3-20A1M showed noticeable ratios of dispensable and specific genes in the signal transduction and cell motility categories (Fig. 3E). In the signal transduction category, the specific genes belonged to a sub-category, the two-component system. Genes related to flagella assembly and bacterial chemotaxis, which are sub-categories of the cell motility category, are also included in the two-component system according to KEGG categorization (Table 2). Erythrobacter sp. 3-20A1M-specific genes belonging to flagella assembly included flgKM, fliMS, and $\operatorname{mot} B$, and bacterial chemotaxis included che $A B R$, fliM, and motB. Although other Erythrobacter species also have genes corresponding to the same KO ID, they were recognized as different gene families in the PGAP analysis. Among the two-component systems that did not correspond to the above two categories, genes related to virulence were included. In particular, Erythrobacter sp. 3-20A1M has three genes involved in exopolysaccharide biosynthesis, epsAPC (F7D01_05380, F7D01_13155, and F7D01_13520), of which epsAP was found to be a unique gene [28]. Erythrobacter sp. 3-20A1M also has the pme (pectinesterase, F7D01_10375) gene, encoding a plant cell wall-degrading enzyme, which was also found in the closest related species, E. lutimaris. Pectinesterase is an enzyme that decomposes pectin polysaccharides of plant cell walls. Regarding the marine ecosystem, pectin is also a component of algal cell walls [29]. These findings suggest a role of these two species as scavengers of pectin-containing organisms.

To further investigate the possible scavenging ability against plant-algae, the virulence factors of the 
A

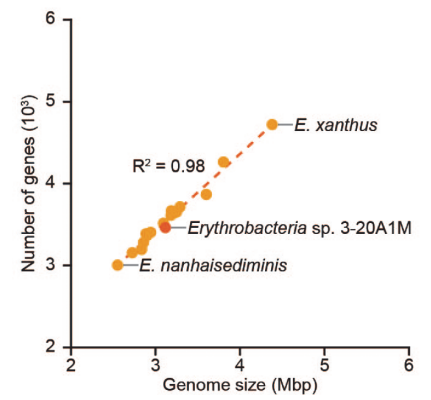

D

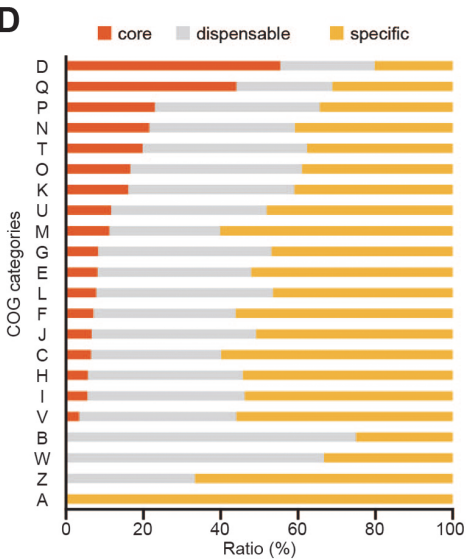

B

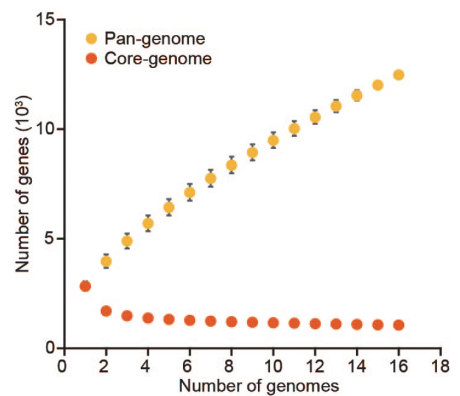

E
C

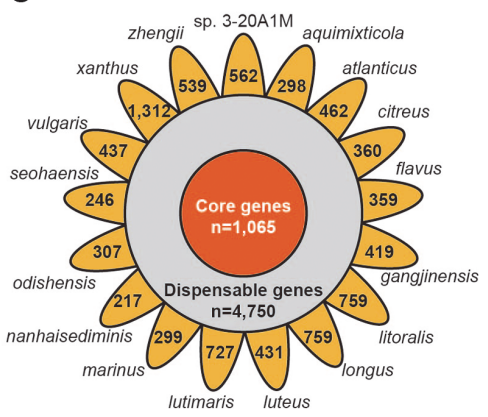

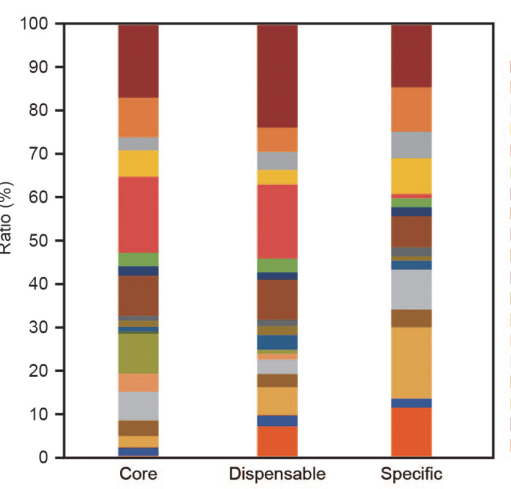

- Carbohydrate metabolism

Lipid metabolism

Nucleotide metabolism

- Amino acid metabolism
-

Metabolism of other amino acids

- Glycan biosynthesis and metabolism

Metabolism of cofactors and vitamins

Metabolism of terpenoids and polyketides

- Xenobiotics biodegradation and metabolism

Transcription

Translation

ing and degradation

Replication and repair
Membrane transport

- Signal transduction

Cellular community - Cell motility

Fig. 3. Pan-genome analysis of Erythrobacter species. A. Correlation between the genome size and the number of coding genes of each genome. B. Pan-genome and core-genome profiles. The number of genes in the Erythrobacter pangenome and core-genome are plotted. C. Pan-Genome analysis pipeline was used to analyze 17 Erythrobacter species. The number of common core genes across all 17 species is presented in the innermost circle (red). The number of dispensible genes is presented in the middle circle (gray). The number of genes specific to each strain is presented in the outmost area (yellow). D. The distribution of core, dispensible, and strain-specific genes presented as a bar graph. COG categories: D, Cell cycle control, cell division, and chromosome partitioning; Q, Secondary metabolites biosynthesis, transport, and catabolism; P, Inorganic ion transport and metabolism; N, Cell motility; T, Signal transduction mechanisms; O, Post-translational modification, protein turnover, and chaperones; K, Transcription; U, Intracellular trafficking, secretion, and vesicular transport; M, Cell wall/ membrane/envelope biogenesis; G, Carbohydrate transport and metabolism; E, Amino acid transport and metabolism; L, Replication, recombination and repair; F, Nucleotide transport and metabolism; J, Translation, ribosomal structure and biogenesis; C, Energy production and conversion; $\mathrm{H}$, Coenzyme transport and metabolism; I, Lipid transport and metabolism; V, Defense mechanisms; B, Chromatin structure and dynamics; W, Extracellular structures; Z, Cytoskeleton; A, RNA processing and modification. E. The KEGG Orthology distribution among core, dispensible, and specific genes of Erythrobacter sp. 3-20A1M.

Erythrobacter sp. 3-20A1M were searched using annotation data and KEGG categories. The virulence genes were searched under four categories: biofilm synthesis, secretion system, motility, and plant-algae cell wall degradation (Table S3). Additional to epsAPC, several exopolysaccharide biosynthetic genes and polysaccharide export genes were found [30]. Erythrobacter sp. 3-20A1M contains three intact bacterial secretion systems, which are the type IV secretion system, Sec-SRP system, and Tat system [31]. The strain contained an intact flagella machinery, which generates motility and also serves as a flagella type III secretion system [32]. By means of identifying the plant-algae cell wall degradation, the genes related to starch metabolism were searched. Along with the pectinesterase, glycosyl hydrolases, pectate lyase, cellulase, and cell wall hydrolase genes were identified that could be used as an arsenal for decomposing plant-algae cell walls.

\section{Secondary Metabolite Biosynthetic Gene Clusters of Erythrobacter Species}

The secondary metabolite, including carotenoid, production capacity of the 17 Erythrobacter species was investigated through secondary metabolite biosynthetic gene cluster (BGC) prediction using the antiSMASH tool [23]. This analysis predicted 58 BGCs across the species (Table S4). Accordingly, the terpene BGC was predicted in all Erythrobacter species. In addition, the types of BGCs that are produced in each species were correlated with their phylogenetic relationships (Fig. 4A). In particular, the clade including E. xanthus, E. luteus, E. odishensis, E. ganginensis, E. aquimixticola, E. atlanticus, E. zhengii, and E. marinus, produces lasso peptide, type 3 polyketide synthetase (T3PKS), and the terpene biosynthetic cluster. Conversely, the lasso peptide and T3PKS were found less frequently in other clades. Interestingly, only E. xanthus was predicted to contain 13 BGCs. However, an error 
Table 2. Erythrobacter sp. specific genes regarding cell motility and signal transduction.

\begin{tabular}{|c|c|c|c|}
\hline \multicolumn{4}{|c|}{ Cell motility } \\
\hline \multicolumn{4}{|c|}{ Flagella assembly } \\
\hline KEGG & Gene ID & Function & Locus tag \\
\hline K02396 & $f \lg K$ & Flagellar hook-associated protein 1 & F7D01_10300 \\
\hline K02398 & $f \lg M$ & Negative regulator of flagellin synthesis & F7D01_10230 \\
\hline K02416 & flim & Flagellar motor switch protein & F7D01_10160 \\
\hline K02422 & flis & Flagellar secretion chaperone & F7D01_10200 \\
\hline K02557 & $\operatorname{mot} B$ & Chemotaxis protein & F7D01_13825 \\
\hline \multicolumn{4}{|c|}{ Bacterial chemotaxis } \\
\hline KEGG & Gene ID & Function & Locus tag \\
\hline K00575 & cheR & Chemotaxis protein methyltransferase & F7D01_01680,F7D01_11905 \\
\hline K02416 & fliM & Flagellar motor switch protein & F7D01_10160 \\
\hline K02557 & $\operatorname{mot} B$ & Chemotaxis protein & F7D01_13825 \\
\hline K03407 & cheA & Chemotaxis sensor kinase & F7D01_11900 \\
\hline K03412 & cheB & Protein-glutamate methylesterase/glutaminase & F7D01_01705, F7D01_01685, F7D01_11910 \\
\hline K13924 & cheR & Chemotaxis protein methyltransferase & F7D01_04690 \\
\hline \multicolumn{4}{|c|}{ Signal transduction } \\
\hline \multicolumn{4}{|c|}{ Two-component system } \\
\hline KEGG & Gene ID & Function & Locus tag \\
\hline K00405 & $\operatorname{ccoO}$ & Cytochrome c oxidase cbb3-type subunit II & F7D01_07745 \\
\hline K00575 & cheR & Chemotaxis protein methyltransferase & F7D01_01680,F7D01_11905 \\
\hline K01051 & pme & Pectinesterase & F7D01_10375 \\
\hline K01104 & epsP & Protein-tyrosine phosphatase & F7D01_13155 \\
\hline K01991 & epsA & Polysaccharide biosynthesis/export protein & F7D01_05380 \\
\hline K02398 & $f l g M$ & Negative regulator of flagellin synthesis & F7D01_10230 \\
\hline K02488 & pleD & Cell cycle response regulator & F7D01_11555 \\
\hline K02659 & pilI & Twitching motility protein PilI & F7D01_01700 \\
\hline K03407 & cheA & Chemotaxis sensor kinase & F7D01_11900 \\
\hline K03412 & cheB & Protein-glutamate methylesterase/glutaminase & F7D01_01705, F7D01_01685, F7D01_11910 \\
\hline K07165 & fecR & Transmembrane sensor & F7D01_09650, F7D01_10520 \\
\hline K07782 & sdiA & Quorum-sensing system regulator & F7D01_01835 \\
\hline K12340 & tolC & Outer membrane protein & F7D01_05315 \\
\hline K13486 & $w s p C$ & Chemotaxis protein methyltransferase & F7D01_02045, F7D01_03085 \\
\hline K13924 & cheR & Chemotaxis protein methyltransferase & F7D01_04690 \\
\hline K18326 & $m d t D$ & Multidrug resistance protein & F7D01_04925 \\
\hline
\end{tabular}

may have caused excessive predictions due to the relatively large number of contigs in the draft genome compared to that of other species, and the PGAP result also showed a large number of unique genes in E. xanthus. To assess the presence of erroneous genome sequences, the genome assembly quality was confirmed using BUSCO, returning a ratio of duplication of $0.7 \%$. It was determined that the overall large number of genes or abundance of BGCs were not caused by duplication errors in the genome assembly process. While Erythrobacter sp. 3-20A1M was not phylogenetically close to the eight species mentioned above, it also produces lasso peptide, T3PKS, terpene, and has a BGC for homoserine lactone production. Among Erythrobacter species, the newly identified Erythrobacter sp. 3-20A1M produces diverse secondary metabolites.

The terpene biosynthetic pathway utilizes either the mevalonate (MVA) pathway or the methylerythritol 4phosphate (MEP) pathway and starts with pyruvate and glyceraldehyde 3-phosphate generated in glycolysis [33]. Most organisms have only one of the pathways, and Erythrobacter species utilize the MEP pathway subsequent isoprenoid pathway to produce a terpenoid backbone precursor called geranyl-geranyl-pyrophosphate, which is used as a precursor of the carotenoid biosynthetic pathway. Next, we compared the terpene biosynthetic gene clusters of the 17 Erythrobacter species according to their functional roles (Fig. 4B). While all the terpene biosynthetic pathway genes were conserved among the 17 species based on KEGG analysis, the terpene BGCs predicted by antiSMASH were diversified. Most Erythrobacter species, including Erythrobacter sp. 3-20A1M, contain a single terpene BGC with two core genes, accessory proteins, and at least one transport gene, whereas E. atlanticus and E. zhengii have multiple terpene BGCs (Fig. 4B).

\section{Carotenoid Biosynthesis Pathway across Erythrobacter Species}

Erythrobacter species are known to produce excess carotenoids [8]. Carotenoid biosynthesis begins with geranyl-geranyl-pyrophosphate as a precursor generated from the terpene biosynthetic pathway (Fig. 5A). From the KEGG analysis, it was found that, except for E. citreus, all the Erythrobacter species studied have conserved pathways for zeaxanthin production from geranyl-geranyl-pyrophosphate with intermediate compounds, such as lycopene and $\beta$-carotene, and astaxanthin pathways to produce astaxanthin from zeaxanthin. However, six 
A

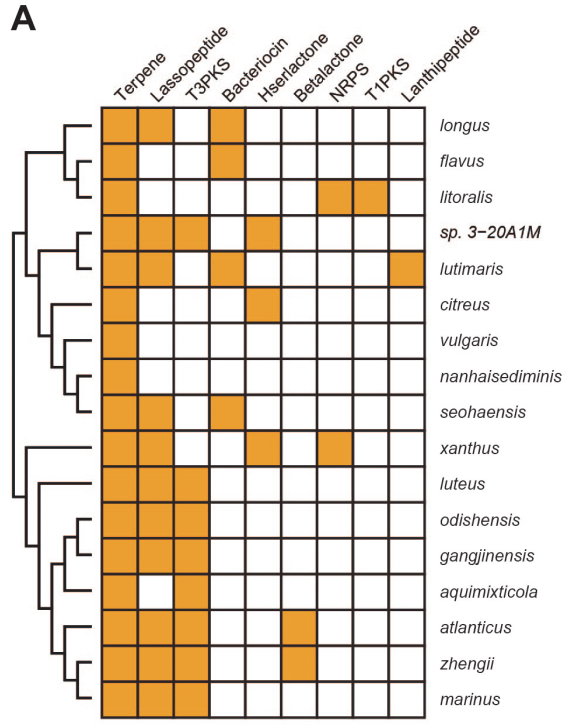

B $\square$ Core $\square$ Additional $\square$ Transport $\square$ Regulatory $\square$ Others

$\square \square$

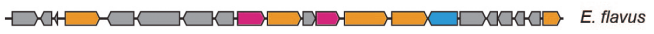

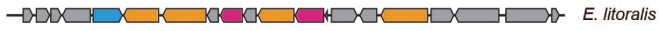
- Enthrobacter sp. 3-20A1M

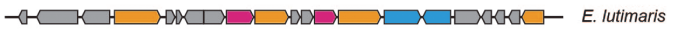
$\mathrm{C} \longrightarrow \mathrm{CO}$

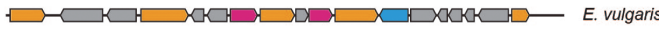

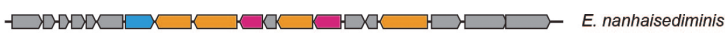

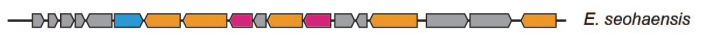
$\longrightarrow$ -

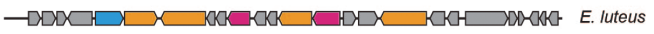

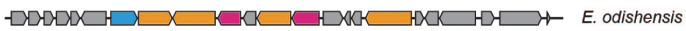

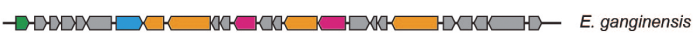

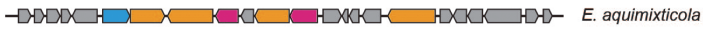
| atlanticus

$\longrightarrow \square \mathrm{CDX} \square \mathrm{WW}=\square \mathrm{CO}$ $\rightarrow \longrightarrow$ E. zhengii (1)

C E. marinus

Fig. 4. Secondary metabolites production of Erythrobacter species. A. Secondary metabolite biosynthetic gene clusters were predicted in 17 Erythrobacter species. Color indicates the presence of the biosynthetic gene cluster. The species were arranged by their phylogenetic relationships. B. Predicted terpene biosynthetic clusters are presented. Abbreviations: T3PKS, type 3 polyketide synthetase; Hserlactone, homoserine lactone; NRPS, non-ribosomal peptide synthase.

A

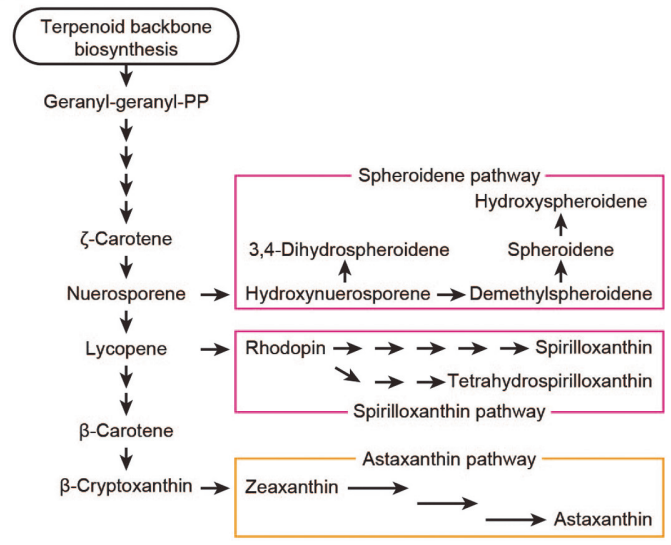

C

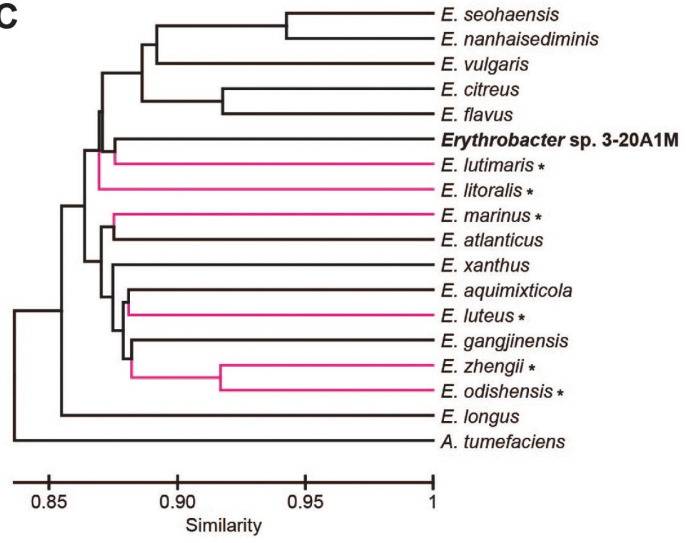

B

E. litoralis

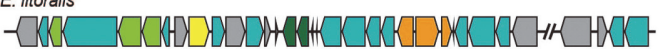

E. longus

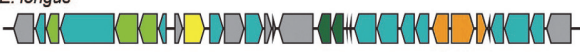

E. Iutimaris

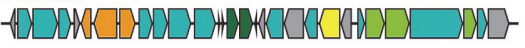

E. marinus

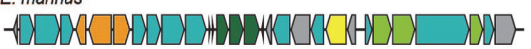

E. odishensis

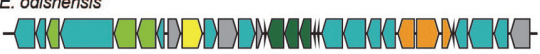

E. zhengii

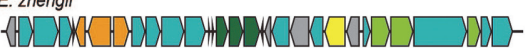

$\square$ bch $\square$ chl $\square$ puf $\square$ crt $\square p p s R$ घothers

Fig. 5. Carotenoid biosynthesis of Erythrobacter species. A. The carotenoid biosynthetic pathway is presented. The astaxanthin, spirilloxanthin, and spheroidene pathways are highlighted. B. Photosynthetic gene clusters are presented in 6 Erythrobacter species. C. Average nucleotide identity-based phylogeny tree of the 17 Erythrobacter species. Aerobic anoxygenic photosynthetic bacteria are highlighted with asterisks and indicated in magenta. 
Erythrobacter species, E. lutimaris, E. litoralis, E. marinus, E. luteus, E. zhengii, and E. odishensis, have additional carotenoid biosynthesis pathways, including the spheroidene pathway and the spirilloxanthin pathway. These two pathways are used for pigment production in photosynthetic bacteria, such as purple bacteria. Erythrobacter species are phylogenetically close to purple sulfur bacteria, and this is the suspected reason for the presence of photosynthetic bacteria among Erythrobacter species $[5,10]$. They are classified according to their photosynthetic ability, and the photosynthetic species are called AAPB. The difference in photosynthetic ability among Erythrobacter species depends on the presence of the PGC. Through the analysis of the KO ID annotations, six Erythrobacter species, E. litoralis, E. longus, E. lutimaris, E. marinus, E. odishensis, and E. zhengii, were found to have both PGC and spheroidene/sprilloxanthin pathways required for the production of photosynthetic pigments (Fig. 5B and Table S5). The discovered PGC includes puf, encoding light-harvesting complex subunits; $b c h$, for bacteriochlorophyll production; chl, for chlorophyll production; and crt, for additional carotenoid biosynthesis. A photosynthesis regulator, $p p s R$, is also present in the cluster and regulates the transcription of gene clusters such as that of bch, crt, puf, and puc [34]. As no PGC or additional carotenoid biosynthetic pathways were found in Erythrobacter sp. 3-20A1M, it is thought to be a heterotrophic bacterium and not an AAPB.

An evolutionary explanation for the lack of PGCs in Erythrobacter sp. 3-20A1M is that the presence of a large gene cluster is more likely to arise from a common ancestor than individual strains experiencing separate gene cluster deletions. However, in the phylogenetic analysis based on the UBCG, the AAPBs with PGCs did not appear to be bound under the common ancestral phylogeny branch (Fig. 1C). Although the UBCG analysis uses more sequences than the 16S rRNA sequence analysis, it remains a comparison using selected sets of genes. Since the absence of a large genetic element, such as a PGC, which is not included in the UBCG, may affect the analysis, phylogeny was re-analyzed by comparing the average nucleotide identity using the whole genome sequences (Fig. 5C) [35]. However, AAPB-possessing species were not grouped under the common ancestral branch, even in whole-genome sequence-based calculations. For example, the non-AAPB strain, Erythrobacter sp. 3-20A1M, is clustered near the AAPB strain, E. lutimaris. A more in-depth evolutionary analysis is needed to determine the evolutionary events that trigger the current distribution of PGCs across Erythrobacter species.

In summary, the complete genome sequence of a recently isolated marine bacterium, Erythrobacter sp. 3$20 \mathrm{~A} 1 \mathrm{M}$, was assembled. Subsequent genotypic and pan-genome analyses were conducted to identify the bacterium. The comparative genomic analysis of the bacterium compared to other Erythrobacter species highlighted its inability to generate energy phototrophically and heterotrophic nature. Additionally, the presence of the virulence factors composed of biofilm generation, secretion system, flagella motility, and plant-algal cell wall degradation suggests its marine environmental niche as an active scavenger.

\section{Acknowledgments}

This work was supported by the Basic Core Technology Development Program for the Oceans and the Polar Regions Korea (2016M1A5A1027455 to S.C., and NRF-2016M1A5A1027458 to B.-K.C), and the Basic Science Research Program (2018R1A1A3A04079196 to S.C.) through the National Research Foundation (NRF), funded by the Ministry of Science and ICT of Korea.

\section{Conflict of Interest}

The authors have no financial conflicts of interest to declare.

\section{References}

1. Worden AZ, Follows MJ, Giovannoni SJ, Wilken S, Zimmerman AE, Keeling PJ. 2015. Rethinking the marine carbon cycle: factoring in the multifarious lifestyles of microbes. Science 347: 1257594

2. Faust K, Raes J. 2012. Microbial interactions: from networks to models. Nat. Rev. Microbiol. 10: 538.

3. Lidicker Jr WZ. 1979. A clarification of interactions in ecological systems. Bioscience 29: 475-477.

4. Kolber ZS, Gerald F, Lang AS, Beatty JT, Blankenship RE, VanDover CL, et al. 2001. Contribution of aerobic photoheterotrophic bacteria to the carbon cycle in the ocean. Science 292: 2492-2495.

5. Zheng Q, Lin W, Liu Y, Chen C, Jiao N. 2016. A comparison of 14 Erythrobacter genomes provides insights into the genomic divergence and scattered distribution of phototrophs. Front. Microbiol. 7: 984.

6. SHIBA T, SIMIDU U. 1982. Erythrobacter longus gen. nov., sp. nov., an aerobic bacterium which contains bacteriochlorophyll $a$. Int. J. Syst. Evol. Microbiol. 32: 211-217.

7. Takaichi S. 2009. Distribution and biosynthesis of carotenoids, pp. 97-117. The Purple Phototrophic Bacteria, Ed. Springer, New York, USA.

8. Takaichi S, Shimada K, Ishidsu J-i. 1990. Carotenoids from the aerobic photosynthetic bacterium, Erythrobacter longus: $\beta$-carotene and its hydroxyl derivatives. Arch. Microbiol. 153: 118-122.

9. Galasso C, Corinaldesi C, Sansone C. 2017. Carotenoids from marine organisms: Biological functions and industrial applications. Antioxidants 6: 96.

10. Yurkov VV, Beatty JT. 1998. Aerobic anoxygenic phototrophic bacteria. Microbiol. Mol. Biol. Rev. 62: 695-724.

11. Breed MF, Harrison PA, Blyth C, Byrne M, Gaget V, Gellie NJ, et al. 2019. The potential of genomics for restoring ecosystems and biodiversity. Nat. Rev. Genet. 20: 615-628.

12. Cho S-H, Lee E, Ko S-R, Jin S, Song Y, Ahn C-Y, et al. 2020. Elucidation of the biosynthetic pathway of Vitamin B groups and potential secondary metabolite gene clusters via genome analysis of a marine bacterium Pseudoruegeria sp. M32A2M. J. Microbiol. Biotechnol. 30: 505-514.

13. Roosaare M, Puustusmaa M, Möls M, Vaher M, Remm M. 2018. PlasmidSeeker: identification of known plasmids from bacterial whole genome sequencing reads. PeerJ. 6: e4588.

14. Na S-I, Kim YO, Yoon S-H, Ha S-m, Baek I, Chun J. 2018. UBCG: Up-to-date bacterial core gene set and pipeline for phylogenomic tree reconstruction. J. Microbiol. 56: 280-285. 
15. Stamatakis A. 2014. RAxML version 8: a tool for phylogenetic analysis and post-analysis of large phylogenies. Bioinformatics 30: $1312-1313$

16. Kumar S, Stecher G, Li M, Knyaz C, Tamura K. 2018. MEGA X: molecular evolutionary genetics analysis across computing platforms. Mol. Biol. Evol. 35: 1547-1549.

17. Tatusova T, DiCuccio M, Badretdin A, Chetvernin V, Nawrocki EP, Zaslavsky L, et al. 2016. NCBI prokaryotic genome annotation pipeline. Nucleic Acids Res. 44: 6614-6624.

18. Kanehisa M, Sato Y, Kawashima M, Furumichi M, Tanabe M. 2015. KEGG as a reference resource for gene and protein annotation. Nucleic Acids Res. 44: D457-D462.

19. Tatusov RL, Galperin MY, Natale DA, Koonin EV. 2000. The COG database: a tool for genome-scale analysis of protein functions and evolution. Nucleic Acids Res. 28: 33-36.

20. Consortium TGO. 2014. Gene Ontology Consortium: going forward. Nucleic Acids Res. 43: D1049-D1056.

21. Muller J, Szklarczyk D, Julien P, Letunic I, Roth A, Kuhn M, et al. 2010. eggNOG v2. 0: extending the evolutionary genealogy of genes with enhanced non-supervised orthologous groups, species and functional annotations. Nucleic Acids Res. 38: D190-D195.

22. Zhao Y, Wu J, Yang J, Sun S, Xiao J, Yu J. 2012. PGAP: pan-genomes analysis pipeline. Bioinformatics 28: 416-418.

23. Blin K, Shaw S, Steinke K, Villebro R, Ziemert N, Lee SY, et al. 2019. antiSMASH 5.0: updates to the secondary metabolite genome mining pipeline. Nucleic Acids Res. 47: W81-W87.

24. Simão FA, Waterhouse RM, Ioannidis P, Kriventseva EV, Zdobnov EM. 2015. BUSCO: assessing genome assembly and annotation completeness with single-copy orthologs. Bioinformatics 31: 3210-3212.

25. Li X, Koblížek M, Feng F, Li Y, Jian J, Zeng Y. 2013. Whole-genome sequence of a freshwater aerobic anoxygenic phototroph, Porphyrobacter sp. strain AAP82, isolated from the Huguangyan Maar Lake in Southern China. Genome Announc. 1: e0007213.

26. Xu X-W, Wu Y-H, Wang C-S, Wang X-G, Oren A, Wu M. 2009. Croceicoccus marinus gen. nov., sp. nov., a yellow-pigmented bacterium from deep-sea sediment, and emended description of the family Erythrobacteraceae. Int. J. Syst. Evol. Microbiol. 59: 22472253.

27. Medini D, Donati C, Tettelin H, Masignani V, Rappuoli R. 2005. The microbial pan-genome. Curr. Opin. Genet. Dev. 15: $589-594$.

28. Dertli E, Mayer MJ, Colquhoun IJ, Narbad A. 2016. EpsA is an essential gene in exopolysaccharide production in Lactobacillus johnsonii FI9785. Microb. Biotechnol. 9: 496-501.

29. Domozych DS, Sørensen I, Popper ZA, Ochs J, Andreas A, Fangel JU, et al. 2014. Pectin metabolism and assembly in the cell wall of the charophyte green alga Penium margaritaceum. Plant Physiol. 165: 105-118.

30. Coleman RJ, Patel YN, Harding NE. 2008. Identification and organization of genes for diutan polysaccharide synthesis from Sphingomonas sp. ATCC 53159. J. Ind. Microbiol. Biotechnol. 35: 263-274.

31. Alvarez-Martinez CE, Christie PJ. 2009. Biological diversity of prokaryotic type IV secretion systems. Microbiol. Mol. Biol. Rev. 73: $775-808$

32. Minamino T. 2018. Hierarchical protein export mechanism of the bacterial flagellar type III protein export apparatus. FEMS Microbiol. Lett. 365: fny117.

33. Oldfield E, Lin FY. 2012. Terpene biosynthesis: modularity rules. Angew. Chem. Int. Ed. 51: 1124-1137.

34. Moskvin OV, Gomelsky L, Gomelsky M. 2005. Transcriptome analysis of the Rhodobacter sphaeroides PpsR regulon: PpsR as a master regulator of photosystem development. J. Bacteriol. 187: 2148-2156.

35. Lee I, Kim YO, Park S-C, Chun J. 2016. OrthoANI: an improved algorithm and software for calculating average nucleotide identity. Int. J. Syst. Evol. Microbiol. 66: 1100-1103. 